\title{
Luminescence-based magnetic imaging with scanning x-ray transmission microscopy
}

\author{
C. A. F. Vaz, ${ }^{1, \text { a) }}$ C. Moutafis, ${ }^{2}$ C. Quitmann, ${ }^{2}$ and J. Raabe ${ }^{2}$ \\ ${ }^{1}$ SwissFEL, Paul Scherrer Institut, 5232 Villigen PSI, Switzerland \\ ${ }^{2}$ Swiss Light Source, Paul Scherrer Institut, 5232 Villigen PSI, Switzerland
}

(Received 27 April 2012; accepted 9 August 2012; published online 23 August 2012)

\begin{abstract}
We demonstrate the imaging of the magnetic domain configuration of cobalt structures fabricated on $\mathrm{MgO}(001)$ using $\mathrm{x}$-ray induced optical luminescence in a scanning transmission microscope. The technique relies on the measurement of the magnetization-dependent $\mathrm{x}$-ray absorption probed by the optical luminescence radiated from the $\mathrm{MgO}$ substrate and induced by the $\mathrm{x}$-rays transmitted through the magnetic layer. This method enables the measurement of the electronic and magnetic spectroscopic properties of single crystalline layers and buried heterostructures with nanometer lateral resolution and elemental sensitivity and opens scanning transmission x-ray microscopy to materials which cannot be grown on membranes or as freestanding thin films. (ㅇ 2012 American Institute of Physics. [http://dx.doi.org/10.1063/1.4747697]
\end{abstract}

The development of characterization techniques capable of probing phenomena down to the nanoscale have been instrumental to the establishment of a paradigm in science whereby the properties of materials are tailored from the bottom-up (nanotechnology). Examples include scanning transmission electron microscopy, scanning tunneling and atomic force microscopies, photoemission electron microscopy, and scanning transmission x-ray microscopy (STXM), which have allowed a deeper understanding of physical phenomena occurring at the interface between dissimilar materials, ${ }^{1-3}$ and the investigation of the static and dynamic behavior of magnetism and ferroelectricity in nanosized elements. ${ }^{4-6} \mathrm{STXM}^{7}$ in particular, is a powerful tool for the investigation of magnetic phenomena in small elements and can be combined with time resolution down to a few 10 ps. ${ }^{89}$ It is based on the measurement of x-rays transmitted through the magnetic material and its dependence on the photon energy and polarization. STXM has been circumscribed for the most part to magnetic layers deposited on $\mathrm{x}$-ray transparent SiN membranes. Although thinning of a substrate is possible, the latter procedure becomes cumbersome when the study of patterned structures is necessary. In this Letter, we report a development in STXM that circumvents the last drawback. It relies on the measurement of the optical luminescence from the substrate induced by the x-rays transmitted through the sample layer. We demonstrate this principle by imaging the magnetic domain structure of patterned cobalt structures. We show that this technique opens STXM for the measurement of single crystalline samples (including buried layers) grown epitaxially on crystalline substrates, with nanometer lateral resolution.

For the experimental set-up, the STXM installed at the PolLux beamline ${ }^{9}$ of the Swiss Light Source (Paul Scherrer Institut, Villigen, Switzerland) was modified to detect the optical luminescence radiating from the substrate onto which a Co layer was deposited. We use a 1:1 image transfer optical

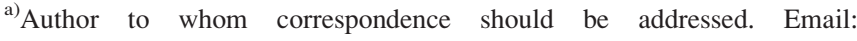
carlos.vaz@cantab.net.
}

line and a charge-coupled device (CCD) camera detector (Andor DV860DCS-BV) with a spectral response in the range from 400 to $900 \mathrm{~nm}$. The set-up is schematically shown in Fig. 1. The CCD camera also provides visual imaging of the sample, facilitating its alignment in the microscope. In STXM, the X-ray beam is focused by a Fresnel zone plate (FZP) to spot sizes down to $15 \mathrm{~nm}$ in diameter. It is the X-ray spot size that provides the spatial resolution of this technique and which in turn enables the use of the luminescence from the substrate as a measure of the x-ray transmission through the sample. The use of luminescence as a measure of the $\mathrm{x}$-ray absorption has been reported previously in the literature, but without spatial resolution. ${ }^{10,11}$ The principle of operation is demonstrated here for a patterned cobalt structure deposited on a $\mathrm{MgO}(001)$ single crystal, which was chosen for its strong luminescence. The polycrystalline Co film, $20 \mathrm{~nm}$ in thickness, was deposited at $300 \mathrm{~K}$ in ultrahigh vacuum (base pressure of $1 \times 10^{-9} \mathrm{mbar}$ ) by thermal evaporation of elemental $\mathrm{Co}$ onto a $\mathrm{MgO}$ substrate (double-side polished), which was shadow-masked by a copper grid to form an array of square elements, $50 \mu \mathrm{m}$ in size. The x-ray attenuation length of $\mathrm{Co}$ is of about $500 \mathrm{~nm}$ at the pre-edge and about $70 \mathrm{~nm}$ at the $L$ edge $(779.7 \mathrm{eV}) .{ }^{12}$ For the measurements shown here, the sample was imaged at room temperature in the as-grown state. Although the $\mathrm{x}$-ray beam was nominally focused to a $25 \mathrm{~nm}$ spot size, the spatial resolution in our measurements was limited to about $100 \mathrm{~nm}$ by the x-ray exit slits, which were widened to increase the beam flux. The imaging resolution also varied, depending on the field of view. To obtain magnetic contrast (proportional to the dot product between the light polarization $\mathbf{P}$ and the magnetization, $\mathbf{M}$ ) for the in-plane magnetized Co elements, the sample normal was tilted by $30^{\circ}$ with respect to the $\mathrm{x}$-ray beam direction $(\mathbf{P})$. The energy spectra were acquired with a step size of $0.1 \mathrm{eV}$ and smoothed with five-point averaging. The resolving power of the PolLux beamtime was set to $\Delta E / E=1000$ and the integration time was typically $10 \mathrm{~ms}$ per pixel. Drifts in the scanned images were corrected using the TurboReg and StackReg routines. ${ }^{13}$ 


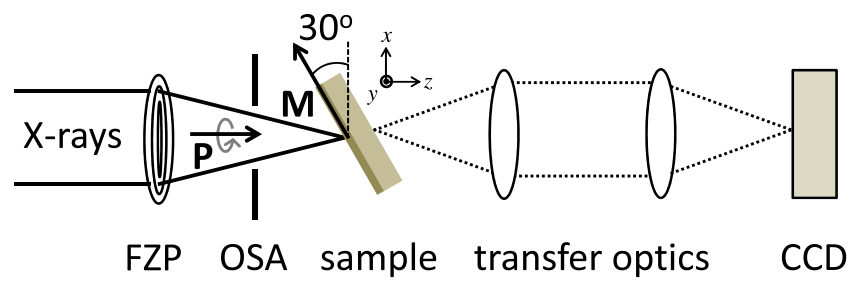

FIG. 1. Schematic diagram of the measurement set-up and sample geometry (FZP: Fresnel zone plate; OSA: order sorting aperture; $\mathbf{P}$ is the light polarization and $\mathbf{M}$ the sample magnetization). The sample consists of a $20 \mathrm{~nm}$ thick Co film grown on a transparent luminescent substrate.

The element sensitivity of photoluminescence-based magnetic imaging is illustrated in Fig. 2, which shows the detected optical luminescence intensity as a function of the sample position $(x, y)$. The dark squares in Fig. 2(a) are due to the stronger $x$-ray absorption of the Co film (the shadows observed in the image are due to a slight shift in the shadow mask during the Co deposition and corresponds to a very thin Co layer). X-ray energy line scans across the dashed line shown in Fig. 2(a) demonstrate the elemental sensitivity of this technique; this is illustrated in Fig. 2(b), which plots the energy scan on a spot (ca. $100 \mathrm{~nm}$ diameter) in the Co square (A), showing a well defined Co $2 p$ absorption edge, which is absent in the scan on a spot on the substrate (B) where no Co has been deposited. This shows that this technique is well suited for spectroscopic characterization of materials with $\mathrm{nm}$ spatial resolution. In particular, it makes films grown on bulk substrates accessible to STXM. We note that although the Co film imaged is polycrystalline, a similar outcome would result for an epitaxial film. In fact, this detection method is potentially more suitable for single crystalline films, which often require bulk single crystal substrates for epitaxial growth. It works best for a film thickness at which the intensity is reduced by $1 / 2$ (or $\ln 2 \times \lambda, \lambda=$ absorption length at the absorption edge) because this gives highest signal to noise ratio (SNR). Using the current set-up, the thinnest films would be about $10 \mathrm{ML}(10 \mathrm{~ms} /$ pixel, SNR $\sim 3)$. This range can be further extended by increasing the integration time (factor of 100 for a reduction in thickness by a factor of 10).

Magnetic contrast is obtained by imaging the sample with left and right circularly polarized light. Representative results are given in Fig. 3(a), which shows the normalized difference between the absorption maps for right $\left(I_{\mathrm{r}}\right)$ and left $\left(I_{1}\right)$ circularly polarized light, $I=\left(I_{\mathrm{r}}-I_{1}\right) /\left(I_{\mathrm{r}}+I_{1}\right) \sim \mathbf{M} \cdot \mathbf{P}$.
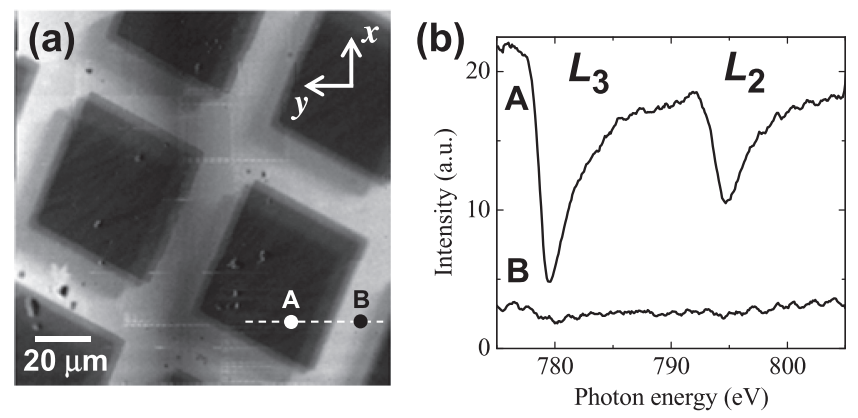

FIG. 2. (a) Scanning x-ray absorption image of a $20 \mathrm{~nm}$ thick Co element array at $779.7 \mathrm{eV}$ detected by photoluminescence. (b) Energy scans across the Co $L$ edge for two positions in the sample as labeled in (a) taken with a focused $\mathrm{x}$-ray beam, $\sim 100 \mathrm{~nm}$ in diameter.
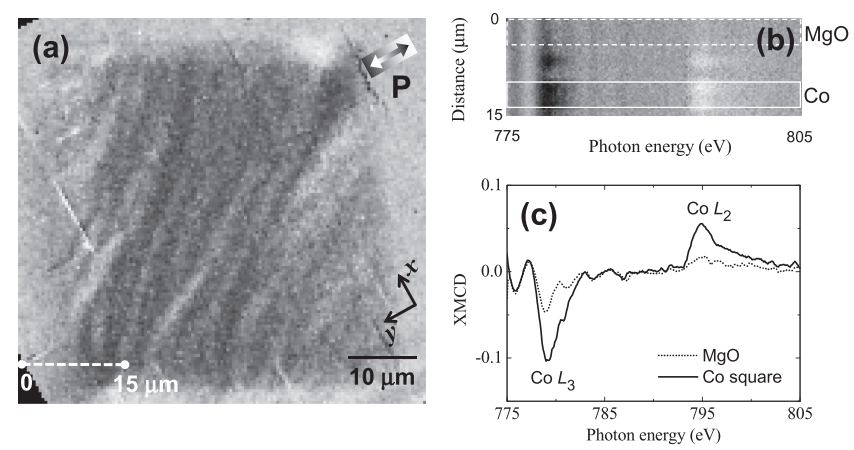

FIG. 3. Scanning X-ray magnetic dichroism images of a $50 \mu \mathrm{m}$ Co square $(779.7 \mathrm{eV})$. (b) X-ray magnetic circular dichroism energy scan for the Co $L$ edge across the dashed line shown in (a); the signal integrated across the full and dashed box is shown in (c) for the $\mathrm{MgO}$ and Co regions of the sample, respectively.

These data demonstrate the suitability of the present method to image magnetic domains in structures grown on thick single crystals. This procedure is particularly advantageous for the characterization of epitaxially grown crystalline structures, including insulating and/or buried layers, which cannot be accessed by techniques relying on electron photoemission to measure light absorption. The magnetic domain structure in the Co square element consists of ripple domains with inplane magnetization and with neighbouring domains pointing in orthogonal directions. The squares are large enough that no magnetic flux-closed states favored by the shape anisotropy are observable in the as-grown state. An energy scan (step size of $150 \mathrm{~nm} /$ pixel) across the Co $L$ edge along the dashed line shown in Fig. 3(a), which includes two magnetic domains, was carried out for both light polarizations and the resulting $\mathrm{X}$-ray magnetic circular dichroic (XMCD) signal is shown in Fig. 3(b). By looking at the XMCD intensity at the $L_{3}$ absorption edge $(779.7 \mathrm{eV})$, one finds that the top region, which lies outside the Co square, shows little XMCD signal (a small contribution arises from residual Co due to a shift in the shadow mask during film deposition); inside the Co square, a strong XMCD signal (black) first appears corresponding to a magnetic domain pointing in the direction of the light polarization, followed by an area of weak contrast (grey), corresponding to a magnetic domain pointing in a direction nearly orthogonal to the light polarization $(\mathbf{M} \perp \mathbf{P})$; a strong XMCD signal appears again further into the Co square. The XMCD signal in the larger domain is shown in Fig. 3(c), corresponding to the average over 30 scans. The good signal to noise ratio demonstrates that quantitative analysis of the magnetic spectroscopic data with spatial resolution down to the nanoscale is possible with this technique.

We consider next the X-ray excited photoluminescence response of $\mathrm{MgO}$. X-ray excited optical luminescence (XEOL) is an X-ray photon in, optical photon out excitation process, where optical photons are generated in the process of de-excitation following absorption of an x-ray photon. ${ }^{14}$ In XEOL, luminescence can be produced by direct excitation as well as by secondary processes, where high energy electrons such as photoelectrons and Auger electrons thermalize in the solid, producing additional secondary electrons and holes. ${ }^{14}$ Luminescence then results from the radiative recombination of low energy electrons that populate the bottom of 


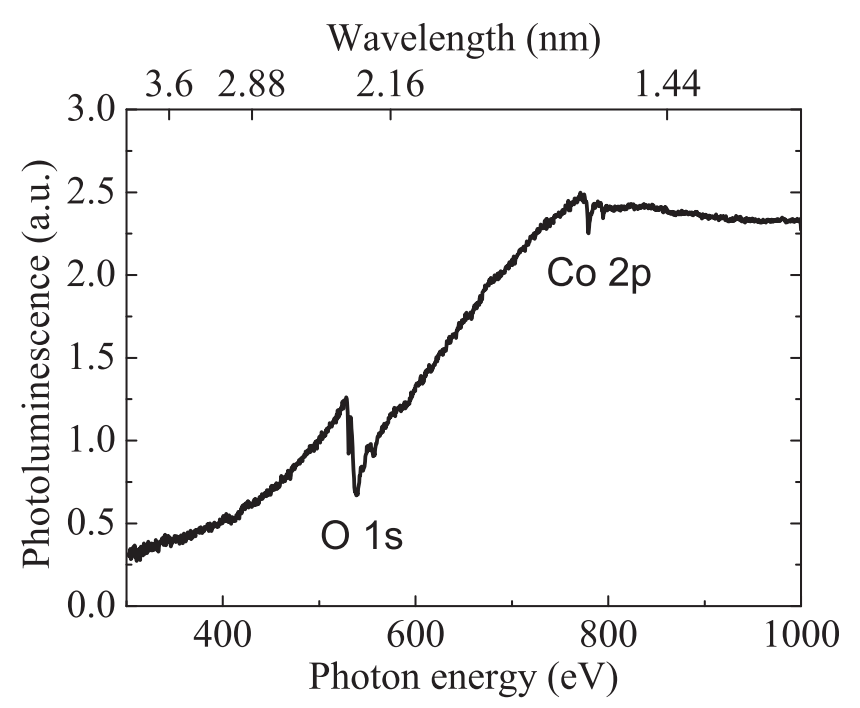

FIG. 4. X-ray induced optical luminescence of $\mathrm{MgO}$ as a function of photon energy. The luminescence signal is integrated over the whole visible range, set by the spectral sensitivity of the CCD detector.

the conduction band and the shallow holes at the top of the valence band. These produce light known as band-gap or near band-gap emission, which is excitonic in nature. ${ }^{15,16}$ The luminescence spectrum of $\mathrm{MgO}$ is characterized by a broad line at around $5.4 \mathrm{eV}(\lambda=230 \mathrm{~nm})$ arising from the recombination of electrons with holes localized at oxygen vacancy centers, and a sharper line at around $7.7 \mathrm{eV}(\lambda=161 \mathrm{~nm})$ due to the radiated decay of free excitons (with a lifetime in the sub-ns range). ${ }^{17,18}$ In Fig. 4, we show the optical luminescence response of $\mathrm{MgO}$ (normalised to the incoming $\mathrm{x}$-ray flux and integrated over the spectral response of the detector) as a function of incident photon energy across the x-ray region that includes the absorption edges of the $3 \mathrm{~d}$ transition metals $(300-1000 \mathrm{eV})$. The $\mathrm{O} 1 \mathrm{~s}$ absorption edge of $\mathrm{MgO}$ is clearly observed at $535 \mathrm{eV}$, together with a small Co contribution at $780 \mathrm{eV}$, which we attribute to imperfect shadowing of the probed area during the Co deposition (see Fig. 2). One observes that the XEOL yield increases in the range from 400 to $800 \mathrm{eV}$ and flattens out above $800 \mathrm{eV}$, implying a higher efficiency for the measurement of higher photon energies or higher atomic number elements.

Here, we have only considered the static response of the luminescence signal. The luminescence spectrum of solids depends strongly on the nature of the band gap (direct or indirect), the presence of charge traps (impurities, lattice defects, oxygen vacancies, or surface states), the density of excited carriers, the energy of the incident light, and temperature; each of these gives rise to specific spectral lines with different lifetimes. By filtering out the long lifetime components (for instance, by using optical filters), it may be possible to add time resolution to the present method. In addition, other substrates merit investigation. For example, preliminary measurements of the $\mathrm{x}$-ray induced photoluminescence of $\mathrm{SrTiO}_{3}$ shows that, although exhibiting a much lower luminescence efficiency compared to $\mathrm{MgO}$, it can also be employed in this technique. Using the short life-time photoluminescence radiation transitions of $\mathrm{MgO}$ or $\mathrm{SrTiO}_{3}$ (ns range, Refs. 19 and 20) will allow the investigation of dynamical phenomena down to the $\mathrm{x}$-ray time resolution of storage rings (10's of ps). Although the present measurement technique is limited to substrates that luminescence, this family includes most insulating oxides such as $\mathrm{MgO}, \mathrm{SrTiO}_{3}, \mathrm{Al}_{2} \mathrm{O}_{3}$, which are widely used for the epitaxial growth of complex oxides, metallic ferromagnets, and Heusler alloys, to mention a few important examples. ${ }^{21,22}$

In summary, we show that the luminescence response of bulk crystals can be utilized to measure the spatially resolved $\mathrm{x}$-ray absorption coefficient of thin films grown on such crystals in STXM. This provides chemical and magnetic information on the film with nanoscale lateral resolution. By using this method, the capabilities of scanning transmission $\mathrm{x}$-ray microscopy can be extended to include the characterization of single crystalline materials with no requirements for specialized sample preparation. In addition, it has the capability of probing buried layers and, in principle, can be readily extended to include time-resolved experiments.

The authors would like to thank F. Nolting for use of the deposition system at the SIM beamline (SLS). Part of this work was performed at the Swiss Light Source, Paul Scherrer Institut, Villigen, Switzerland.

${ }^{1}$ R. A. McKee, F. J. Walker, and M. F. Chisholm, Science 293, 468 (2001).

${ }^{2}$ D. A. Muller, Nature Mater. 8, 263 (2009).

${ }^{3}$ J. Garcia-Barriocanal, F. Y. Bruno, A. Rivera-Calzada, Z. Sefrioui, N. M. Nemes, M. Garcia-Hernández, J. Rubio-Zuazo, G. R. Castro, M. Varela, S. J. Pennycook, C. Leon, and J. Santamaria, Adv. Mater. 22, 627 (2010).

${ }^{4}$ J. Raabe, C. Quitmann, C. H. Back, F. Nolting, S. Johnson, and C. Buehler, Phys. Rev. Lett. 94, 217204 (2005).

${ }^{5}$ C. V. K. Schmising, M. Bargheer, M. Kiel, N. Zhavoronkov, M. Woerner, T. Elsaesser, I. Vrejoiu, D. Hesse, and M. Alexe, Phys. Rev. Lett. 98, 257601 (2007).

${ }^{6}$ D. Su, Q. Meng, C. A. F. Vaz, M.-G. Han, Y. Segal, F. J. Walker, M. Sawicki, C. Broadbridge, and C. H. Ahn, Appl. Phys. Lett. 99, 102902 (2011).

${ }^{7}$ B. Kaulich, P. Thibault, A. Gianoncelli, and M. Kiskinova, J. Phys.: Condens. Matter 23, 083002 (2011).

${ }^{8}$ P. Fischer, T. Eimüller, G. Schütz, G. Denbeaux, A. Pearson, L. Johnson, D. Attwood, S. Tsunashima, M. Kumazawa, N. Takagi, M. Köhler, and G. Bayreuther, Rev. Sci. Instrum. 72, 2322 (2001).

${ }^{9}$ J. Raabe, G. Tzvetkov, U. Flechsig, M. Böge, A. Jaggi, B. Sarafimov, M. G. C. Vernooij, T. Huthwelker, H. Ade, D. Kilcoyne, T. Tyliszczak, R. H. Fink, and C. Quitmann, Rev. Sci. Instrum. 79, 113704 (2008).

${ }^{10}$ G. Jakob, T. Eichhorn, M. Kallmayer, and H. J. Elmers, Phys. Rev. B 76, 174407 (2007).

${ }^{11}$ M. Meinert, J.-M. Schmalhorst, G. Reiss, and E. Arenholz, J. Phys. D: Appl. Phys. 44, 215003 (2011).

${ }^{12}$ B. L. Henke, E. M. Gullikson, and J. C. Davis, At. Data Nucl. Data Tables 54, 181 (1993).

${ }^{13}$ P. Thévenaz, U. E. Ruttimann, and M. Unser, IEEE Trans. Image Process. 7, 27 (1998).

${ }^{14}$ J. Y. P. Ko, F. Heigl, Y. M. Yiu, X.-T. Zhou, T. Regier, R. I. R. Blyth, and T.-K. Sham, Can. J. Chem. 85, 853 (2007).

${ }^{15}$ L. Soderholm, G. K. Liu, M. R. Antonio, and F. W. Lytle, J. Chem. Phys. 109, 6745 (1998).

${ }^{16}$ L. Armelao, F. Heigl, S. Brunet, R. Sammynaiken, T. Regier, R. I. R. Blyth, L. Zuin, R. Sankari, J. Vogt, and T.-K. Sham, ChemPhysChem 11, 3625 (2010).

${ }^{17}$ G. H. Rosenblatt, M. W. Rowe, J. G. P. Williams, R. T. Williams, and Y. Chen, Phys. Rev. B 39, 10309 (1989).

${ }^{18}$ M. Kirm, A. Lushchik, C. Lushchik, S. Vielhauer, and G. Zimmerer, J. Lumin. 102-103, 307 (2003).

${ }^{19}$ A. Rubano, D. Paparo, F. M. Granozio, U. S. di Uccio, and L. Marrucci, J. Appl. Phys. 106, 103515 (2009).

${ }^{20}$ H. Yasuda and Y. Kanemitsu, Phys. Rev. B 77, 193202 (2008).

${ }^{21}$ T. Graf, C. Felser, and S. S. P. Parkin, Prog. Solid State Chem. 39, 1 (2011).

${ }^{22}$ M. Opel, J. Phys. D: Appl. Phys. 45, 033001 (2012). 